\title{
Using of a snap-through truss absorber in the attenuation of the sommerfeld effect
}

\author{
W.R.A. de Godoy ${ }^{1}$, J.M.Balthazar ${ }^{2}$, B.R. Pontes Jr. ${ }^{1}$, and J.L.P. Felix ${ }^{3}$ \\ ${ }^{1}$ UNESP, Department of Mechanics Engineering(FEB),Universidade Estadual Paulista, Brazil \\ ${ }^{2}$ UNESP, Department of Statistics, Applied Mathematics and Computation (DEMAC), Universidade Estadual Paulista, \\ Brazil \\ ${ }^{3}$ UNIPAMPA, Federal University of Pampa, Brazil
}

\begin{abstract}
This work, considers a vibrating system, which consists of a snap-through truss absorber (STTA) coupled to an oscillator, under excitation of an DC motor, with an eccentricity and limited power, characterizing a non-ideal oscillator (NIO). It is aimed to use the absorber STTA, to establish the conditions, that we have the maxim attenuation of the jumpphenomenon (Sommerfeld Effect). Here, weare interestedin determining the conditions of the vibrating system, in which there arereduced amplitudes of the oscillator, when it passes through the region of resonance.
\end{abstract}

\section{Introduction}

This work, considers a non-ideal vibrating system, which is characterized by the mutual interaction between the system's response and the excitation. The response influences system's excitation, in opposite to the traditional one, called ideal systems. The non-ideal systems theories can be seenin details in: [1] and[2], undeserving of others authors.

Here, we used a vibrating system, formed by an oscillating block and a DC motor, with limited power, which works as an excitation source. This situation, characterizes a non-ideal system. The passage through resonance reveals interesting behaviors, once the motor is able to transfer a big part of its energy to carry out system oscillations, generating large movement's amplitude. Thereby, we used a DC motor, as a model of linear torque. This torque was used as a control parameter, in order to obtain the passage through resonance and control motor's frequency.

The main phenomenon observed in non-ideal systems is the called Sommerfeld effect, in which the oscillator presents unstable movements at resonance regions. A jump can be seen in the frequency-response curve, revealing the conditions where there is no permanent state.

In this work, we used the snap-through trussSTTA, in the absorption of the longitudinal vibrations of a nonideal oscillating system. Here, the oscillation energy of the main system is transferred to STTA, which fluctuates around an equilibrium point. The analysis ofthe free oscillation of this system was recently studied by [3].
Later, the forced system coupled STTA, was studied by [4]. Also, in recent work [5]analyzed the interaction between STTA and an elastic system.

Also, two previous works, $[6,7]$ analysed the use of STTA in attenuation of the jump phenomenon.

Thegoal of this paper is to analyse the phenomenon of the mutual interaction, between the non-ideal oscillator and the STTA, so that the vibration amplitudes are reduced in the passage through resonance and the jump phenomenon (Sommerfeld effect) is attenuated.

This paper is organized as the follows:Section 2 presents the adopted mathematical model, whichrepresents the non-ideal system connected to a STTA. In Section 3, the stability analysis is exhibited. In Section 4, results and numerical simulations are showed and in Section 5, conclusions will be presented. Finally, we list the bibliographic references

\section{Mathematical model}

The system considered here, is based onan extension of the previous works, from[3 - 6], and may be described by figure 1. In this research, we will aim to show: How the coupling of a small snap-through truss mass, can absorb part of the non-ideal system vibrations, considering especially the passage through resonance and the Sommerfeld effect [1]. 


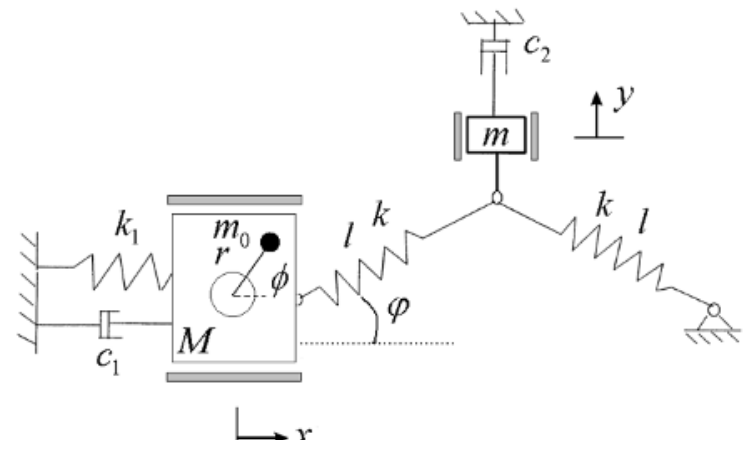

Fig. 1.A non-ideal structure attachment, coupled to snapthrough truss absorber.

The governing equations of motion, which represent the mathematical model of the non-ideal oscillator coupled to snap-through truss absorber, are shown below:

$$
\begin{aligned}
& m_{1} \ddot{x}+2 k\left[x-l \cos \varphi+\frac{l(l \cos \varphi-x) l^{2}}{\sqrt{l^{2}+2 l(y \sin \varphi-x \cos \varphi)+x^{2}+y^{2}}}\right] \\
& =-k_{1} x+m_{0} r\left(\dot{\phi}^{2} \sin \phi-\ddot{\phi} \cos \phi\right)-c_{1} \dot{x} \\
& I \ddot{\phi}=\Gamma(\dot{\phi})-m_{0} r \ddot{x} \cos \phi \\
& m \ddot{y}+2 k\left[y+l \sin \varphi-\frac{l(l \sin \varphi+y)}{\sqrt{l^{2}+2 l(y \sin \varphi-x \cos \varphi)+x^{2}+y^{2}}}\right] \\
& =-c_{2} \dot{y}
\end{aligned}
$$

where $(x, y, \phi)$ are the generalized coordinates of NIO, STTA and rotor, respectively. $(\dot{\phi})$ is the angular velocity of the rotor and $(\phi)$ is the rotation angle of the DC motor shaft. $(M, m)$ are theNIO mass and STTA mass, respectively. $\left(r, m_{0}\right)$ are the eccentricity and unbalanced mass of the electric motor considered. $I$ is the moment of inertia of the rotor. $\left(k, k_{1}\right)$ are the linear stiffness of the springs. $\left(c_{1}, c_{2}\right)$ are NIO and STTA linear damping. Finally, $l$ is the spring length and $\varphi$ is the angle that defines STTA equilibrium position. By using a linear torque model, we consider that $\Gamma(\dot{\phi})=u_{1}-u_{2} \dot{\phi}$, in which $u_{1}$ is related to the voltage applied to the motor and acts as a control parameter for our problem, and $u_{2}$ is constant for each type of motor.

\subsection{Dimensionless system}

Using the following dimensionless parameters:

$$
\begin{aligned}
& \omega_{n}=\sqrt{\frac{k_{1}}{M+m_{0}}}, \tau=\omega_{n} t, u=\frac{x}{l}, v=\frac{y}{l}, \eta_{1}=\frac{m_{0} r}{\left(M+m_{0}\right) l}, \\
& \eta_{2}=\frac{m_{0} r l}{I}, \alpha_{1}=\frac{c_{1}}{\left(M+m_{0}\right) \omega_{n}}, \alpha_{2}=\frac{c_{2}}{m \omega_{n}}, \gamma=\frac{k}{k_{1}}, \\
& \mu=\frac{M+m_{0}}{m}, \Gamma\left(\varphi^{\prime}\right)=a-b \varphi^{\prime}, a=\frac{u_{1}}{I \omega_{n}^{2}}, b=\frac{u_{2}}{I \omega_{n}}, c=\cos \varphi, \\
& s=\sin \varphi
\end{aligned}
$$

We can write the governing equations of motion,as:

$$
\begin{aligned}
& u^{\prime \prime}+u+2 \gamma(u-c) K(u, v)=\eta_{1}\left(\phi^{\prime 2} \sin \phi-\phi^{\prime \prime} \cos \phi\right)-\alpha_{1} u^{\prime} \\
& \phi^{\prime \prime}=a-b \phi^{\prime}-\eta_{2} u^{\prime \prime} \cos \phi \\
& v^{\prime \prime}+2 \mu \gamma(s+v) K(u, v)=-\alpha_{2} v^{\prime}
\end{aligned}
$$

In which

$$
K(u, v)=1-\frac{1}{\sqrt{1+2(v s-u c)+u^{2}+v^{2}}}
$$

\section{Equilibrium points and stability}

Defining new variables, we will write the system in the space state.

$$
\begin{aligned}
& x_{1}=u, x_{2}=u^{\prime}, x_{3}=v, x_{4}=v^{\prime}, x_{5}=\phi, x_{6}=\phi^{\prime} \\
& x_{1}^{\prime}=x_{2} \\
& x_{2}^{\prime}=\frac{1}{\Delta}\left[-x_{1}-2 \gamma\left(x_{1}-c\right) K\left(x_{1}, x_{3}\right)\right. \\
& \left.+q_{1} x_{6}^{2} \sin x_{5}-\alpha_{1} x_{2}-q_{1}\left(a-b x_{6}\right) \cos x_{5}\right] \\
& x_{3}^{\prime}=x_{4} \\
& x_{4}^{\prime}=-2 \mu \gamma\left(s+x_{3}\right) K\left(x_{1}, x_{3}\right)-\alpha_{2} x_{4} \\
& x_{5}^{\prime}=x_{6} \\
& x_{6}^{\prime}=\frac{1}{\Delta}\left\{\left(a-b x_{6}\right)-q_{2}\left[-x_{1}-2 \gamma\left(x_{1}-c\right) K\left(x_{1}, x_{3}\right)\right.\right. \\
& \left.\left.+q_{1} x_{6}^{2} \sin x_{5}-\alpha_{1} x_{2}\right] \cos x_{5}\right\} \\
& \Delta\left(x_{1}, x_{3}\right)=1-\frac{1}{\sqrt{1+2\left(x_{3} s-x_{1} c\right)+x_{1}^{2}+x_{3}^{2}}} \\
& \Delta=1-q_{1} q_{2} \cos ^{2} x_{5} \neq 0
\end{aligned}
$$

Equilibrium points:

Now, in (7) we will find the equilibrium points, making $x_{1}^{\prime}=x_{2}^{\prime}=x_{3}^{\prime}=x_{4}^{\prime}=x_{5}^{\prime}=x_{6}^{\prime}=0$.

If $\Delta \neq 0$, we can consider $a=0$. We also assume that $x_{5}=0$ and we will obtain $x_{2}=0, x_{4}=0, x_{6}=0$ .Thus, the equilibrium points are obtained in the following cases:

a) If $x_{1}=0, K\left(x_{1}, x_{3}\right)=0: 2 x_{3} s+x_{3}^{2}=0$

First equilibrium point: $x_{1}=0, x_{2}=0, x_{3}=0, x_{4}=0, x_{5}$ $=0, x_{6}=0$; 
Second equilibrium point: $x_{1}=0, x_{2}=0, x_{3}=-2 s, x_{4}=$ $0, x_{5}=0, x_{6}=0$

b) If $x_{3}=-s$ in (10): $-s+2 \gamma(-s-c) K\left(-s, x_{3}\right)=0$

Third equilibrium point: $x_{1}=\frac{2 \gamma(c-1)}{1+2 \gamma}, x_{2}=0, x_{3}=-s, x_{4}=$ $0, x_{5}=0, x_{6}=0$;

Fourth equilibrium point: $x_{1}=\frac{2 \gamma(c+1)}{1+2 \gamma}, x_{2}=0, x_{3}=-s, x_{4}$ $=0, x_{5}=0, x_{6}=0$

The stability of equilibrium points can be checked using of the Jacobian matrix (13).

$$
J=\left[\begin{array}{cccccc}
0 & 1 & 0 & 0 & 0 & 0 \\
J_{21} & J_{22} & J_{23} & 0 & J_{25} & J_{26} \\
0 & 0 & 0 & 1 & 0 & 0 \\
J_{41} & 0 & J_{43} & -\alpha_{2} & 0 & 0 \\
0 & 0 & 0 & 0 & 0 & 1 \\
J_{61} & J_{62} & J_{63} & 0 & J_{65} & J_{66}
\end{array}\right]
$$

where:

$$
\begin{aligned}
& J_{21}=\frac{1}{\Delta}\left(-1-2 \gamma K-2 \gamma\left(x_{1}-c\right)\left(-c+x_{1}\right)\left(1+2\left(x_{3} s-x_{1} c\right)+x_{1}^{2}+x_{3}^{2}\right)^{-3 / 2}\right) \\
& J_{22}=-\frac{\alpha_{1}}{\Delta}
\end{aligned}
$$$$
J_{23}=\frac{-\gamma\left(x_{1}-c\right)\left(2 s+2 x_{3}\right)}{\left(1+2\left(x_{3} s-x_{1} c\right)+x_{1}^{2}+x_{3}^{2}\right)^{-3 / 2}}
$$$$
J_{25}=\left(q_{1} x_{6}^{2} \cos x_{5}+q_{1}\left(a-b x_{6}\right) \sin x_{5}\right) \Delta^{-1}
$$$$
-\left(g-q_{1}\left(a-b x_{6}\right) \cos x_{5}\right)\left(q_{1} q_{2} \sin 2 x_{5}\right) \Delta^{-2}
$$$$
J_{26}=\frac{1}{\Delta}\left(2 q_{1} x_{6} \sin x_{5}+q_{1} b \cos x_{5}\right)
$$$$
J_{41}=\frac{-2 \mu \gamma\left(s+x_{3}\right)\left(-c+x_{1}\right)}{\left(1+2\left(x_{3} s-x_{1} c\right)+x_{1}^{2}+x_{3}^{2}\right)^{3 / 2}}
$$$$
J_{43}=-2 \mu \gamma K-\frac{2 \mu \gamma\left(s+x_{3}\right)\left(s+x_{3}\right)}{\left(1+2\left(x_{3} s-x_{1} c\right)+x_{1}^{2}+x_{3}^{2}\right)^{3 / 2}}
$$$$
J_{61}=\frac{1}{\Delta}\left\{-q_{2} \cos x_{5}\left[-1-2 \gamma K-2 \gamma\left(x_{1}-c\right)\left(-c+x_{1}\right)\right.\right.
$$$$
\left.\left.\times\left(1+2\left(x_{3} s-x_{1} c\right)+x_{1}^{2}+x_{3}^{2}\right)^{-3 / 2}\right]\right\}
$$$$
J_{62}=\frac{\alpha_{1} q_{2} \cos x_{5}}{\Delta}
$$

$\left.J_{63}=\frac{1}{\Delta}\left(-q_{2} \cos x_{5}\left(-2 \gamma\left(x_{1}-c\right)\left(s+x_{3}\right)\left(1+2\left(x_{3} s-x_{1} c\right)+x_{1}^{2}+x_{3}^{2}\right)^{-3 / 2}\right)\right)\right)$

$$
J_{65}=\left(g q_{2} \sin x_{5}-q_{1} q_{2} x_{6}^{2} \cos ^{2} x_{5}\right) \Delta^{-1}
$$

$-\left(a-b x_{6}-f q_{2} \cos x_{5}\right) q_{1} q_{2} \sin 2 x_{5} \Delta^{-2}$

$J_{66}=\frac{1}{\Delta}\left(-b-q_{1} q_{2} x_{6} \sin 2 x_{5}\right)$

where :

$$
g=-x_{1}-2 \gamma\left(x_{1}-c\right) K+q_{1} x_{6}^{2} \sin x_{5}-\alpha_{1} x_{2}
$$

Now, using the numerical values to the parameters exhibited on table 1, we evaluated the eigenvalues at each equilibrium point and then we will find the stable points of the system (the corresponding eigenvalues must have negative real parts to ensure stability).

Thus, for system (table1), we will obtain:

\section{System:}

$x_{1}=0, x_{2}=0, x_{3}=0, x_{4}=0, x_{5}=0, x_{6}=0 ; \Rightarrow \mathrm{STABLE}$

$x_{1}=\frac{2 \gamma(c-1)}{1+2 \gamma}, x_{2}=0, x_{3}=-s, x_{4}=0, x_{5}=0, x_{6}=0 ;=>$ STABLE

Table 1.Dimensionless parameters of the non-ideal system [6].

\begin{tabular}{|c|c|c|}
\hline Variables & Symbol & $\begin{array}{c}\text { Values } \\
\text { system }\end{array}$ \\
\hline Interaction coefficients & $\eta_{1}, \eta_{2}$ & $0.05,0.35$ \\
\hline Damping coefficients & $\alpha_{1}, \alpha_{2}$ & $0.01,0.03$ \\
\hline Stiffness coefficient & $\gamma$ & 1.0 \\
\hline Mass coefficient & $\mu$ & 100 \\
\hline Angle & $\varphi$ & 0.405 \\
\hline \multicolumn{2}{|r}{}
\end{tabular}

\section{Numerical simulations results and discussionsof the obtained results}

In this paper, numerical simulations were carried out by using Matlab®, with the numerical integrator ode113, Adams-Bashforth-Moulton PECE solver algorithm with variable step-length. Values $\mathrm{s}=0.39$ and $\mathrm{c}=0.92$ were obtained from $\varphi$.

We will consider the non-ideal oscillator (NIO) coupling to STTA. In this case, we will investigate the dynamicbehaviour of the considered system, when the STTA is coupled to it and also we will verify the reduction of amplitudesof the motion, compared to the system without STTA (we are going to analyse how the system works before, during and after passing through the resonance region).

\subsection{Non-ideal vibrating system (NIO)}

Now, considering the case of the non-ideal oscillator (NIO), we will determine through the figure2: what is the input value of the torque of the DC motor $(a)$, in which the jump phenomenon (Sommerfeld effect) occurs, without coupling the STTA to the NIO (black point).

Figure 2, displays the jump phenomenon present in the NIO, with and without coupling, with STTA. In the coupled system, different values for the stiffness parameter $(\gamma)$ were used, in order to analyze the influence of this parameter on the system (considering the other values in table 1). In this figure, we used $\gamma=0.05$ (gray point), $\gamma_{1}=0.35$ (red point), $\gamma_{2}=0.50$ (blue point), $\gamma_{3}=$ 0.70 (green point), $\gamma_{4}=1.0$ (magenta point). For all cases analyzed, the initial conditions are taken nulls.

Figure 2a, shows the maximal amplitudefor each "averaging" value of the angular velocity of considered DC motor. Figure $2 b$, shows the maximal amplitudes versus the control parameter $(a)$. We notice that $\Delta a=$ 
0.01, refers to the step length, in the parameter control. We can see in both figures $2 a$ and $2 b$, that the maximum amplitude and the jump of the frequency of the NIO without coupling occur, when the $(a)$ is close to 1.8 and $\left(\phi^{\prime}\right)$ to 1.0. What we can also confirm when NIO is coupled to STTA, is that while the value of $(\gamma)$ is increased, the maximum amplitudes of the oscillator tend to decrease to $(a)$, value close to 1.8 , region of the jump of NIO. Another aspect tobe verifiedin this figureis the effectthat the change invalue $(\gamma)$ causes to thecurves shown.It iseasy to see thatas we increasethe value of $(\gamma)$, the curves shift to the right, indicating that the jump occurs for values of $(a)$ above1.8. For the frequency or angular velocity $\left(\phi^{\prime}\right)$ the same happens, so the valuesend up being larger than1.0 for the jump.
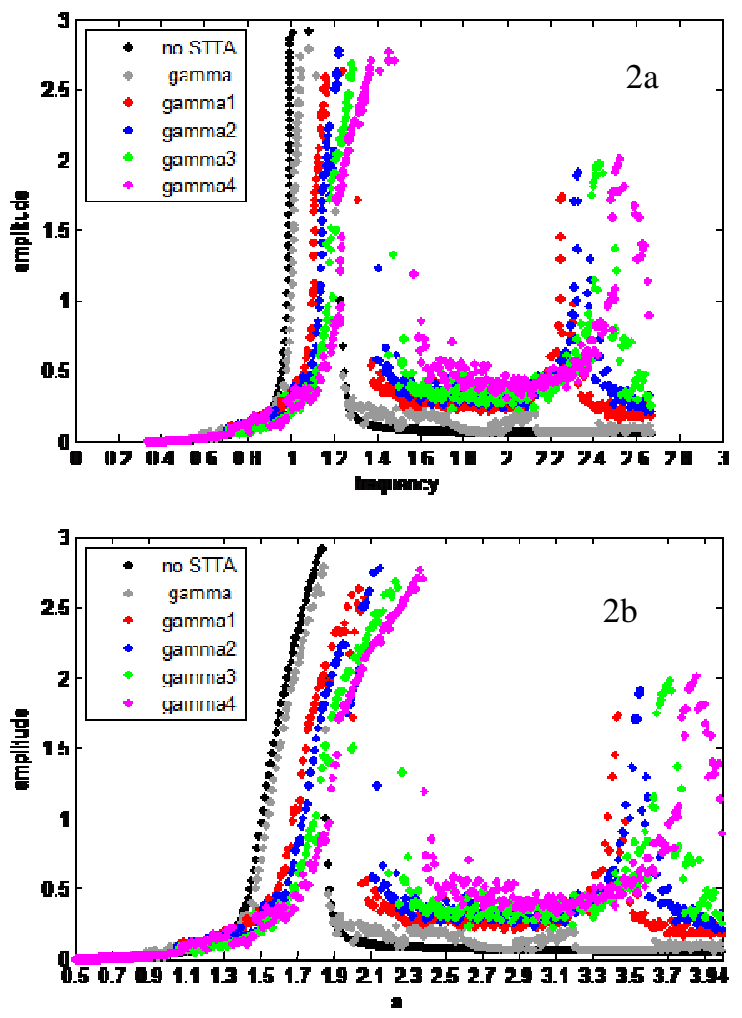

Fig. 4. Sommerfeld effect with $0 \leq a \leq 3.5$ and $\Delta a=0.01$ NIO coupled to STTA: $\gamma=0.05$ (gray point), $\gamma_{1}=0.35$ (red point), $\gamma_{2}=0.50$ (blue point), $\gamma_{3}=0.70$ (green point), $\gamma_{4}=1.0$ (magenta point). NIO uncoupled: black point).

After determining the points, where the jump phenomenon occurs, the behavior of the NIO with and without the STTA is then analyzed. To check the action of the NIO ,in the passage through resonance, we will observe the displacement and angular velocity of the DC motor.

The displacement of the NIO and angular velocity of the motor are shown in figure3, in three different situations: ( $a=1.4)$ before the resonance $(a=1.8)$ in the region of resonance and $(a=2.2)$ after the region. The parameters used are the same as in table 1 . In figure3, we note that when the NIO is coupled to STTA, the angular velocity tends to fluctuate less and maintain a constant value. Also, for the displacement of the NIO, we find that the range of motion is reduced when we have the STTA active in the system.

In figure 3, we note that: before entering of the resonance region (figure $3 \mathrm{a}$ ) and within this region (figure $3 b$ ), the angular velocity tends to fluctuate much less and maintain a constant value when we have the system with the STTA.

The comparisonbetween the displacementsalso revealsthat theoscillations can bedramaticallydecreased, whenthe absorberis attached to theNIO. Since the output of the resonance region (figure $3 \mathrm{c}$ ), the system without coupling oscillates less, while the coupled system is in his own region of resonance, with large fluctuations, as reflected in the figure 2 .
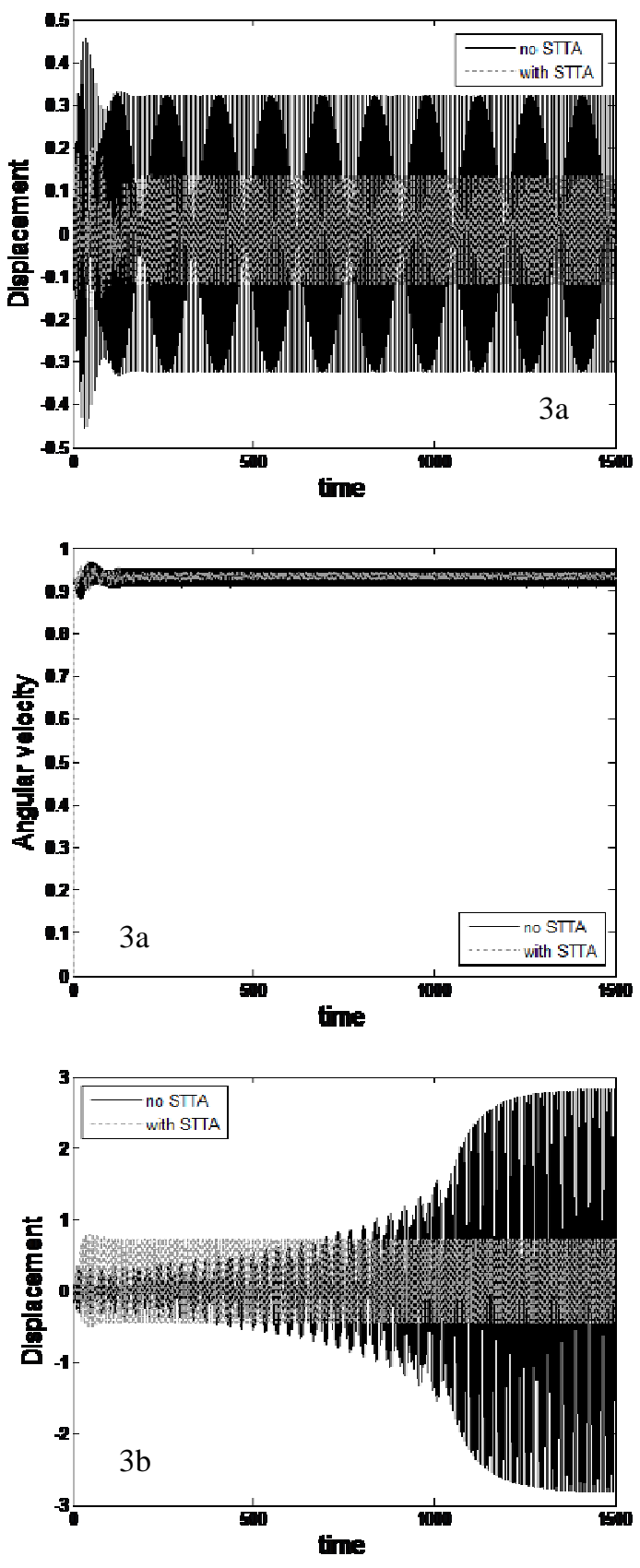

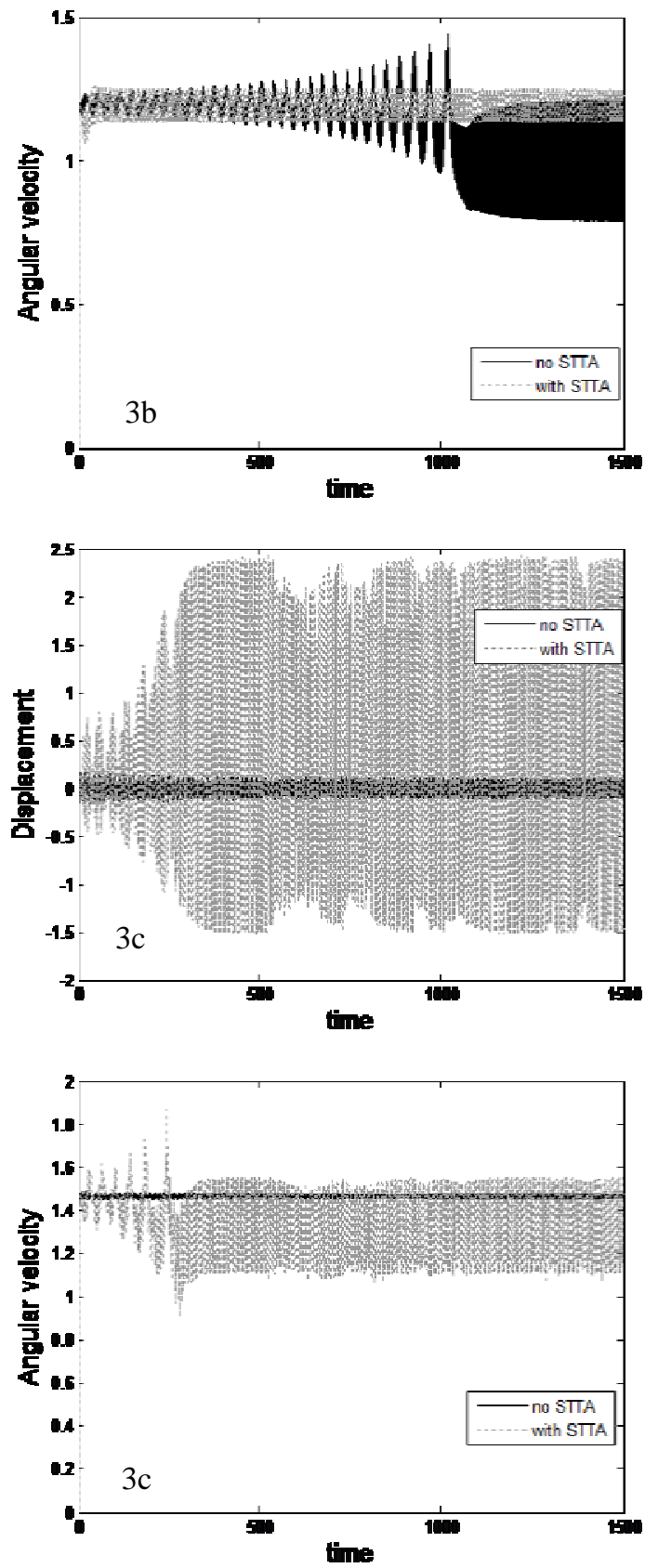

Fig.3. Response of NIO displacement and the angular velocity of motor with coupling (gray line) and without coupling (black line) with (a) $a=1.4$; (b) $a=1.8$; (c) $a=2.2$.

Then, the influence of the stiffness parameter over NIO displacement is observed. Using different values for $(\gamma)$, the time histories are shown when: $(a=1.4)$ before the resonance (figure $4 a),(a=1.8)$ in the resonance region (figure $4 \mathrm{~b})$ and $(a=2.2)$ after the resonance (figure 4c). In these three figures, the displacement corresponding to each value of $(\gamma)$ is shown at each time interval700. The values adopted are: $\gamma=0.05, \gamma_{1}=0.35$, $\gamma_{2}=0.50, \gamma_{3}=0.70, \gamma_{4}=1.0$.
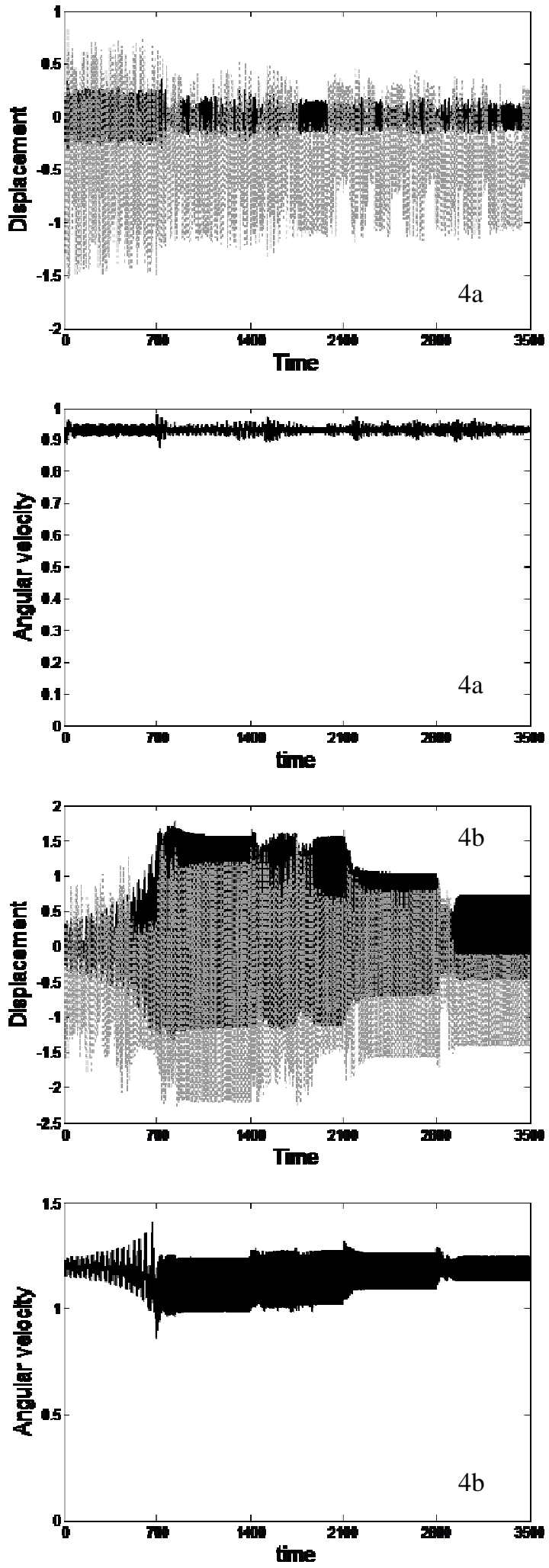

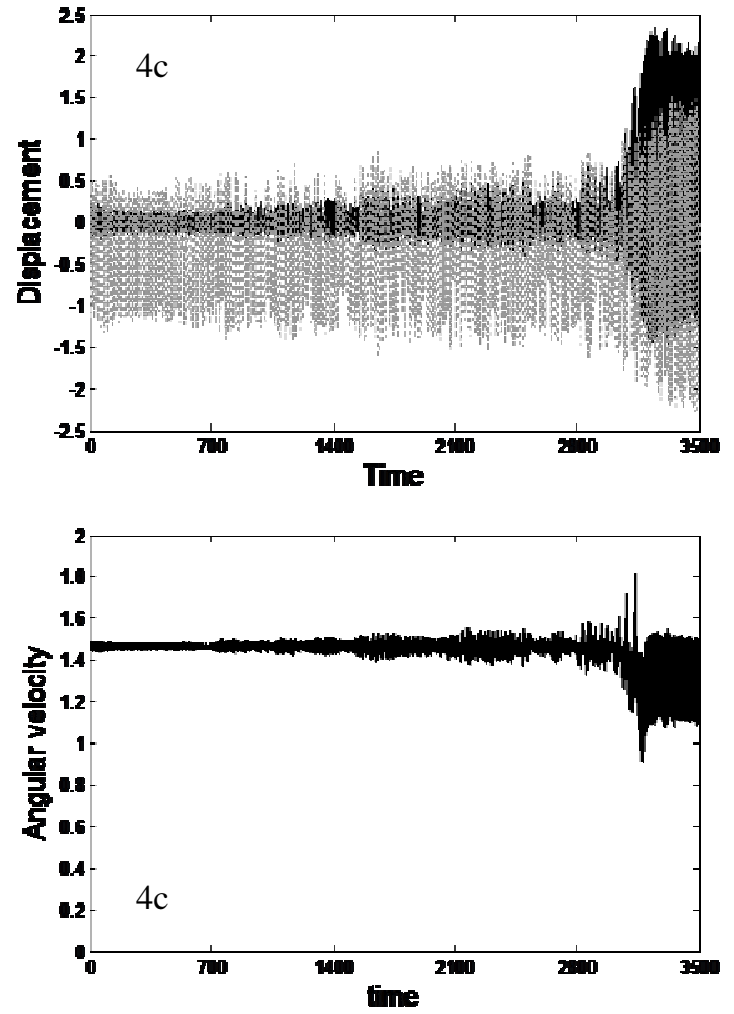

Fig. 4. Time histories for stiffness coefficients with $\gamma=0.05$ in $0 \leq \tau \leq 700, \gamma=0.35$ in $700 \leq \tau \leq 1400, \gamma=0.50$ in $1400 \leq \tau \leq$ $2100, \gamma=0.70$ in $2100 \leq \tau \leq 2800$ and $\gamma=1.0$ in $2800 \leq \tau \leq$ 3500: Responses of NIO (black line) and STTA (gray line). a) $a$ $=1.4$. b) $a=1.8$. c) $a=2.2$.

Looking at figure $4 \mathrm{a}$ and figure $4 \mathrm{~b}$, it is evident that as the coefficient of rigidity increases, the movement amplitude of the NIO, tends to be lower than before. At the same time, the angular velocity also decreases, due to the increased stiffness. However, in figure $4 c$, the increased stiffness, causes that the displacement and angular velocity increase, this is due to the fact, that with this variation of $(\gamma)$ and $(a)=2.2$, the system ends up in the resonance region, a fact that we can verifyby looking atfigure2.

The results of this section show that when we work with NIO, into the resonance region only, the STTA is highly efficient to reduce the amplitudes.

\section{Conclusions}

The non-ideal oscillator, coupled to the STTA proposed in this work, has shown that this type of passive controller is very effective.

The main interest of this application in non-ideal systems is the reduction of the vibration amplitudes, especially when the excitation frequency gets closer to the natural frequency of the system, which creates the jump phenomenon.

Therefore, the absorber generated the aimed results by reducing of effectively the amplitudes of this region.

In future works, starting from the parameters of mass and stiffness, we will try to set the conditions in which occurs the highest energy transfer from the oscillator to the absorber (Energy Pumping phenomenon[8]).

\section{Acknowledgments}

The authors thank FAPESP, CNPq and CAPES, Brazilian financial agencies.

\section{References}

1. Balthazar, J.M., Mook, D.T., Weber, H.I., Brasil, R.M.L.R.F., Fenili, A., Belato, D., Felix, J.L.P., An overview on non-ideal vibrations, Meccanica, 38, No. 6, 613-621 (2003)

2. Nayfeh, A.H. and Mook, D.T., Nonlinear Oscillations. Wiley, New York(1979)

3. Avramov, K. V. and Mikhlin, Yu. V., Snap-through truss as a vibration absorber, Journal of Vibration and Control10, 291-308 (2004)

4. Avramov, K. V. and Mikhlin, Yu. V., Snap-through truss as an absorber of forced oscillations, Journal of Sound and Vibrations 290, 705-722 (2006)

5. Avramov, K. V. and Gendelman, O. V., Interaction of elastic system with snap-through vibration absorber, International Journal of Non-Linear Mechanics 44, 81-89 (2009)

6. Felix . J.L. and Balthazar, J. M., On a Nonlinear Dynamics of a Non-Ideal Oscillator, with a SnapThrough Truss Absorber(STTA), 20th International Congress of Mechanical Engineering. (2009)

7. Godoy, W. R. A., Balthazar, J. M., Pontes Jr, B. R., Felix, J. L.P., On a reduction ofthe Sommerfeld Effect, in a non-ideal vibrating problem, using a snap-through truss passive controller, submitted, (2012).

8. Vakakis, A. F.and Gendelman, O.,Energy pumping in nonlinear mechanical oscillators II: resonance capture. Journal of Applied Mechanics68(1), 42-48 (2001). 